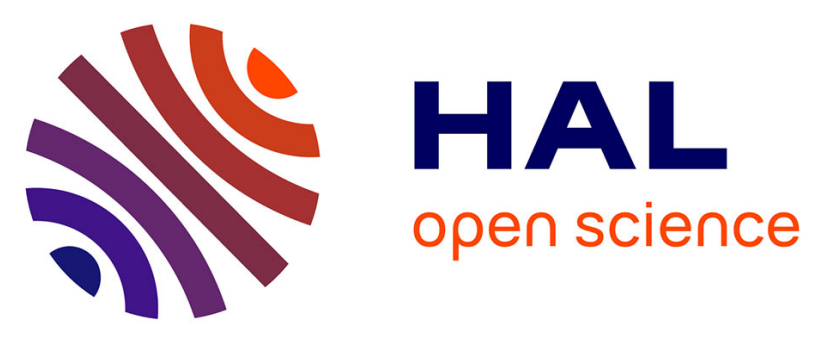

\title{
Supercontinuum generation by nanosecond dual-pumping near the two zero-dispersion wavelengths of a photonic crystal fiber
}

A. Boucon, T. Sylvestre, K. Phan Huy, J.-C. Beugnot, G. Melin, H. Maillotte, J.M. Dudley

\section{To cite this version:}

A. Boucon, T. Sylvestre, K. Phan Huy, J.-C. Beugnot, G. Melin, et al.. Supercontinuum generation by nanosecond dual-pumping near the two zero-dispersion wavelengths of a photonic crystal fiber. Optics Communications, 2011, 284 (1), pp.467-470. 10.1016/j.optcom.2010.09.035 . hal-00582983

\section{HAL Id: hal-00582983 https://hal.science/hal-00582983}

Submitted on 29 Apr 2021

HAL is a multi-disciplinary open access archive for the deposit and dissemination of scientific research documents, whether they are published or not. The documents may come from teaching and research institutions in France or abroad, or from public or private research centers.
L'archive ouverte pluridisciplinaire HAL, est destinée au dépôt et à la diffusion de documents scientifiques de niveau recherche, publiés ou non, émanant des établissements d'enseignement et de recherche français ou étrangers, des laboratoires publics ou privés.

\section{(c)(1)}

Distributed under a Creative Commons Attribution| 4.0 International License 


\title{
Supercontinuum generation by nanosecond dual-pumping near the two zero-dispersion wavelengths of a photonic crystal fiber
}

\author{
Anne Boucon ${ }^{\mathrm{a}, *}$, Thibaut Sylvestre ${ }^{\mathrm{a}}$, Kien Phan Huy ${ }^{\mathrm{a}}$, Jean-Charles Beugnot ${ }^{\mathrm{a}}$, Gilles Mélin ${ }^{\mathrm{b}}$, \\ Hervé Maillotte ${ }^{a}$, John M. Dudley ${ }^{a}$ \\ a Institut FEMTO-ST, Département d'Optique P. M. Duffieux, Université de Franche-Comté, CNRS UMR 6174, 25030 Besançon, France \\ b DRAKA, 91460 Marcoussis, France
}

Supercontinuum generation by dual-wavelength nanosecond pumping in the vicinity of both zero-dispersion wavelengths of a photonic crystal fiber (PCF) is experimentally demonstrated. It is shown in particular that two pumps at $1535 \mathrm{~nm}$ and $767 \mathrm{~nm}$ simultaneously pumping near the two zero-dispersion wavelengths of a specially designed PCF yields a combined visible and infrared supercontinuum spectrum spanning from $0.55 \mu \mathrm{m}$ to $1.9 \mu \mathrm{m}$. We discuss the generation mechanisms underlying the continuum formation in terms of modulation instability and cascaded Raman generation.

\section{Introduction}

The study of supercontinuum (SC) generation in photonic crystal or microstructured fibers continues to be an area of active research, motivated by new applications requiring high intensity light sources over extended wavelength ranges [1-4]. Although many previous reports on SC generation have used fibers with only one zerodispersion wavelength (ZDW), studies of SC generation in photonic crystal fiber (PCF) presenting two ZDWs have been the subject of increasing interest [5-11]. For instance, enhanced SC bandwidth with improved flatness has been demonstrated in PCFs by generating two dispersive waves in the short- and long-wavelength sides of the SC spectrum $[7,10]$. In addition, it has recently been shown that multiwavelength pumping also allows for significant enhancement of the SC bandwidth [12-16]. In this paper, we extend these studies of dual wavelength SC generation by reporting experiments where we have performed dual-wavelength nanosecond pumping, simultaneously exciting broadband SC generation about each ZDW of a specially designed PCF. We use a microchip nanosecond Q-switched laser source at $1535 \mathrm{~nm}$ and periodically poled lithium niobate for efficient frequency doubling at $767 \mathrm{~nm}$, and a PCF with ZDWs at $1540 \mathrm{~nm}$ and $863 \mathrm{~nm}$. The two pumps at $1535 \mathrm{~nm}$ and $767 \mathrm{~nm}$ appear to generate visible and infrared supercontinuua independently, and the combined

\footnotetext{
* Corresponding author. Currently in Laboratoire Interdisciplinaire CARNOT de Bourgogne (ICB), Département OMR, UMR CNRS 5209, Université de Bourgogne, Faculté des Sciences Mirande, 9 Avenue Alain Savary, BP 47 870, 21078 Dijon Cedex, France.

E-mail address: anne.boucon@u-bourgogne.fr (A. Boucon).
}

spectrum spans from $0.55 \mu \mathrm{m}$ to $1.9 \mu \mathrm{m}$. By tailoring the relative power of the two pumps, the spectral content of the spectrum can be significantly varied, and this setup represents a particularly simple and compact means of generating broadband spectra in the visible and infrared spectral regions.

\section{Experimental setup}

The experimental setup is shown schematically in Fig. 1. As a pump source, we use a Cobolt Tango ${ }^{\mathrm{TM}}$ eye safe laser. This pulsed laser emits at $1535 \mathrm{~nm}$ with a repetition rate of $3.3 \mathrm{kHz}$ and an average power of $14 \mathrm{~mW}$, pulse length of $3 \mathrm{~ns}$ and peak power around $1.4 \mathrm{~kW}$. The pump is frequency doubled to $767.5 \mathrm{~nm}$ using a $1 \mathrm{~cm}$ long periodically poled Lithium niobate (PPLN) crystal with a lens of $50 \mathrm{~mm}$ focal length. Owing to the high peak power of the pump laser, other spectral components are also generated in the PPLN crystal such as the fourth harmonic at $384 \mathrm{~nm}$ and a green component at $511 \mathrm{~nm}$ resulting from sum frequency generation (SFG) between the fundamental and the second harmonic [18]. The mean power of the second harmonic component has been estimated at $1.5 \mathrm{~mW}$. Simultaneous coupling of the visible and infrared pumps was achieved for SC generation by using an aspheric lens with infrared coating with an effective focal length of $4.5 \mathrm{~mm}$ in order to minimize coupling loss due to chromatic aberrations. The white-light optical mode at the end of the fiber is also shown in Fig. 1, together with the visible part of the SC spectrum. Fig. 2(a) shows the cross-section (SEM image) of the PCF used. The fiber geometry consists of four rings of holes with different diameters. The first two rings are based on a triangular lattice and the smaller hole diameter is $800 \mathrm{~nm}$. The two external rings consist of 12 larger 


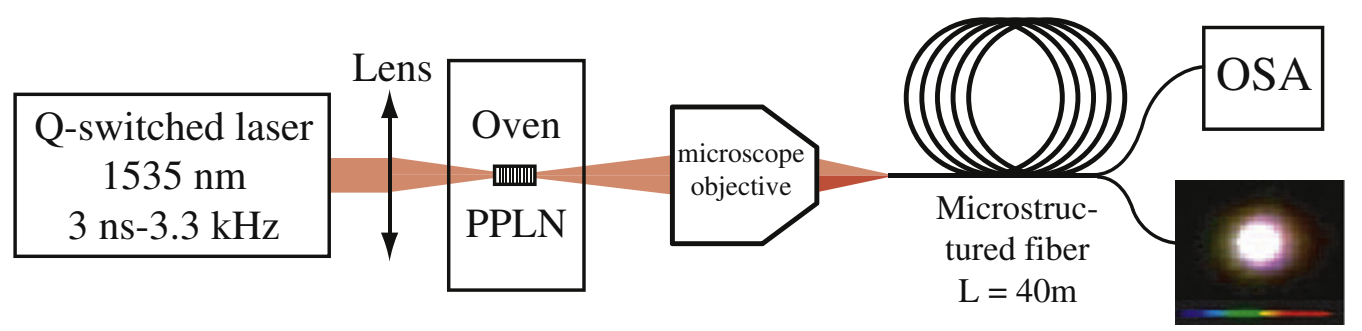

Fig. 1. Experimental setup for supercontinuum generation by dual-wavelength pumping near the two zero-dispersion wavelengths of a photonic crystal fiber.

elliptical holes that isolate the fiber core from the coating to lower the confinement losses. From this SEM image we simulated the fundamental optical mode at the pump wavelength propagating in the fiber using COMSOLC software. The fiber cross-section was imported from the SEM image and the optical mode was solved with the RF module. Fig. 2(b) shows that the fundamental mode is confined inside the first two rings leading to a small effective mode area $A_{e f f}=5 \mu \mathrm{m}^{2}$ and therefore to a high nonlinear coefficient of $\gamma=2 \pi n_{2} /$ $\left(\lambda A_{\text {eff }}\right)=20.5 \mathrm{~W}^{-1} \mathrm{~km}^{-1}$, with $n_{2}=2.5 \times 10^{-20} \mathrm{~m}^{2} \mathrm{~W}^{-1}$. The group velocity dispersion (GVD) curve is also plotted in Fig. 2(c). We clearly see the existence of two zero-dispersion wavelengths located respectively at $863 \mathrm{~nm}$ and $1540 \mathrm{~nm}$.

The two superposed red lines in the figure show the position of two pump wavelengths used for SC generation. Note that the infrared
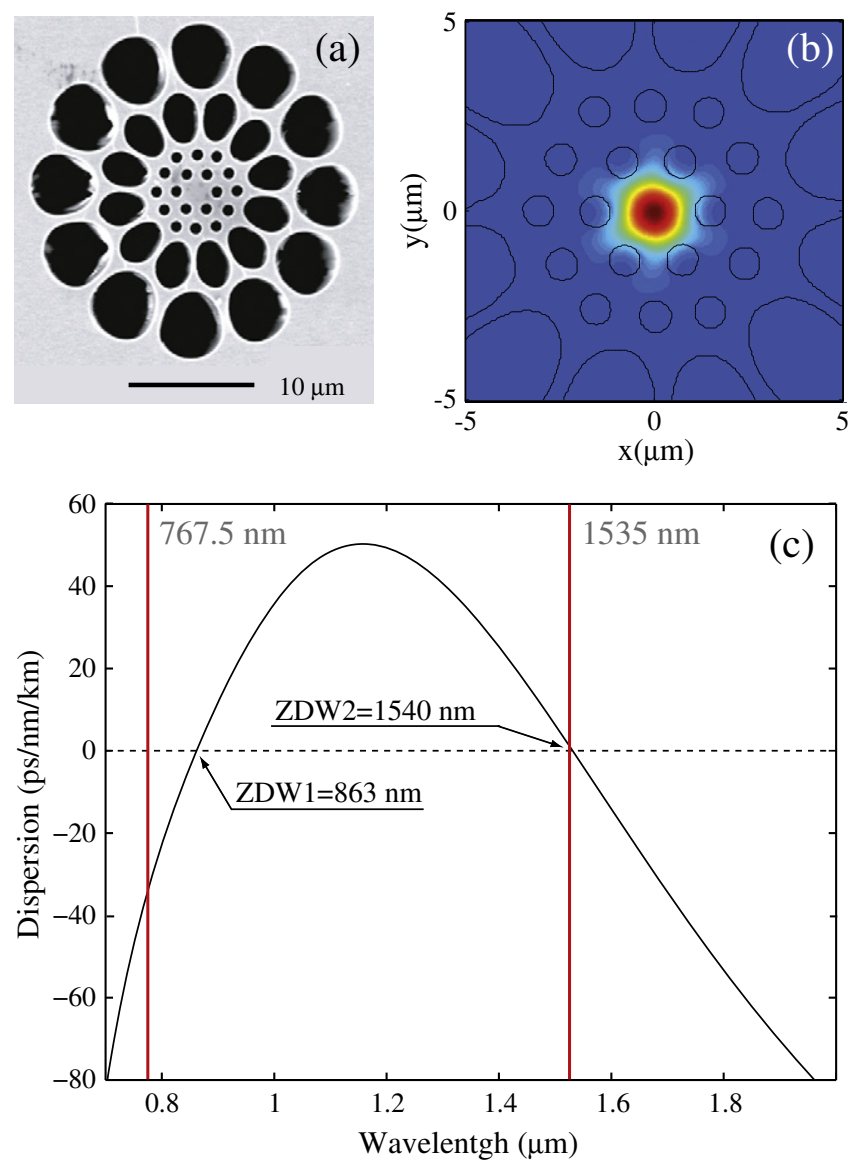

Fig. 2. (a) scanning electronic microscope (SEM) image of the PCF cross section. (b) numerical simulations of the fundamental optical mode confined inside the two first rows of the air-hole microstructure. (c) group-velocity dispersion curve for the fundamental optical mode exhibiting two zero-dispersion wavelengths at $863 \mathrm{~nm}$ and $1540 \mathrm{~nm}$. The vertical red lines indicate the dual pump wavelengths close to the two zero-dispersion wavelengths. pump at $1535 \mathrm{~nm}$ is very close to the second ZDW whereas the visible pump falls slightly in the normal dispersion regime below the first ZDW. It is important to stress here that this situation is significantly different from previous studies of SC generation with two ZDW PCF where the pump wavelengths were always very far from the ZDWs. The second, third and fourth order dispersion coefficients calculated at the pump wavelength of $1535 \mathrm{~nm}$ are $\beta_{2}=1.207 \mathrm{ps}^{2} . \mathrm{km}^{-1}$, $\beta_{3}=-0.315 \mathrm{ps}^{3} . \mathrm{km}^{-1}$ and $\beta_{4}=0.002 \mathrm{ps}^{4} . \mathrm{km}^{-1}$, respectively. Note that the dispersion slope at $1535 \mathrm{~nm}$ is negative and thus leads to a novel regime for infrared SC generation $[2,10,17,19]$.

\section{Experimental results}

We first investigated the general variation in the spectral properties observed as the relative pump-SH power was varied. Fig. 3 shows a false color representation of the measured output spectra for variable coupling efficiency of both infrared and visible pumps. This is achieved simply by translating the aspherical lens along the optical z-axis. Unfortunately, it was not possible to measure the coupling efficiency and the powers launched in the PCF. An estimation was however possible from the spectra and is given in Figs. 4 and 6, that show some output spectra of the color map indicated by horizontal dashed lines in Fig. 3. As seen in Fig. 4, the infrared pump near the second ZDW undergoes spectral broadening through modulation instability (MI), which is manifested, in the Fourier domain, by the clear generation of two sidebands symmetrically located around the pump, as indicated by the arrows in Fig. 4. Their positions in the SC spectrum are predicted by the following phase-matching relation: $\beta_{2} \Omega^{2}+\frac{\beta_{4}}{12} \Omega^{4}+2 \gamma P=0$, $P$ is the infrared peak power and $\Omega=\omega_{M I}-\omega_{P}$ is the frequency shift between the pump and the instability bands. A theoretical model in good agreement with measurements was already presented in details

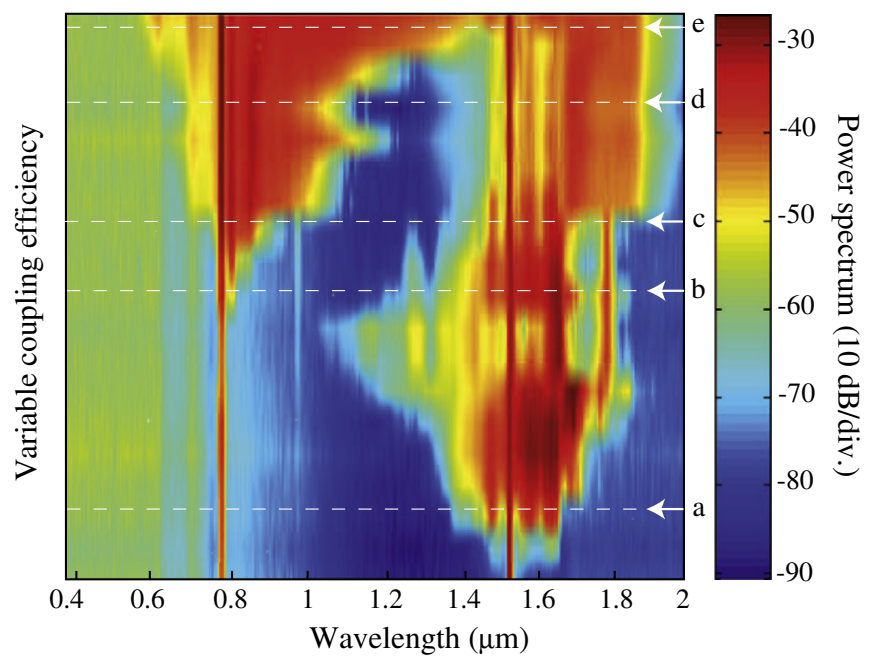

Fig. 3. Color map of output spectra recorded at the end of the photonic crystal fiber for variable coupling efficiency between the infrared to the visible pumps. 


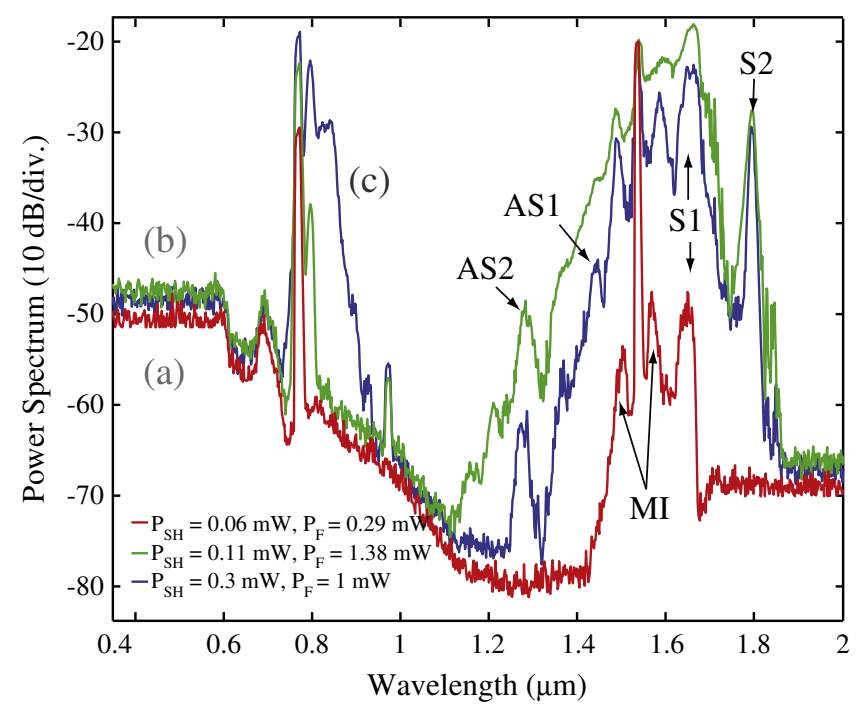

Fig. 4. Output spectra plotted from Fig. 3 associated with a, b, c horizontal dotted lines showing the SC dynamics in the infrared. $\mathrm{P}_{\mathrm{SH}}$ and $\mathrm{P}_{\mathrm{F}}$ are the estimated mean power of the second harmonic and fundamental components. ASi and Si represent respectively the $\mathrm{i}^{\text {th }}$ Raman order of the Anti-Stokes and Stokes components respectively. MI denotes the two modulation instability sidebands.

in Ref. [17]. Stimulated Raman scattering (SRS) also plays a central role in the infrared SC generation and manifests itself through the appearance of a Stokes band which is frequency down-shifted by approximately $13.2 \mathrm{THz}$ from the pump. Fig. 4 shows the generation of a first Raman order denoted S1, strongly seeded by the MI process, and one can also notice the appearance, for sufficiently large pump power, of a second Raman order S2 at $1792 \mathrm{~nm}$. Such a Raman cascade can be clearly observed because the Stokes bands fall within the normal dispersion regime and do not undergo spectral broadening or soliton self-frequency shift dynamics, as it is generally the case when pumping near the first ZDW [19]. Unlike the MI process, the Raman gain is anti-symmetric and spectral components of the antiStokes side, generated by four-wave mixing, have in principle a lower amplitude than those of the Stokes side. However, the observation of anti-Stokes Raman components (AS1, AS2), as shown in Fig. 4, is due to the strong coupling between SRS and parametric gain [17].

The spectral broadening of all anti-Stokes Raman order can be attributed to the fact that they fall in the anomalous dispersion regime and therefore are modulationally unstable.

When we move away the injection lens along the $z$-axis, the coupling efficiency for the infrared pump decreases whereas it increases for the visible pump which is, in turn, spectrally broadened. Fig. 6 shows that the visible pump at $767.5 \mathrm{~nm}$ generates down-frequency-shifted Stokes Raman components through cascading process in the normal dispersion regime of the PCF. An additional spectrum with a higher resolution actually shows that the Raman cascade exhibits four orders as shown in Fig. 5.

As the fourth Raman order falls in the anomalous dispersion regime at $874 \mathrm{~nm}$ just above the first ZDWs of the PCF, SC dynamics then takes place through MI, solitons and dispersive wave generation towards shorter wavelengths, as shown in the spectra plotted in Fig. 6. For higher visible and infrared pump powers, the spectrum in red in Fig. 6 shows that the two independently generated visible and infrared SC spectra merge together to give rise to a wide SC spectrum. The XPM contribution in the SC generation can be however seen in the color map in Fig. 3 when both the visible and IR spectrum exhibit a homogeneous spectral broadening towards longer wavelength. The IR spectrum shows in particular a sharp transition from the second order S2 to a broad and smooth spectrum due of the effect of XPM with the visible part of the spectrum. Moreover, this also yields to the

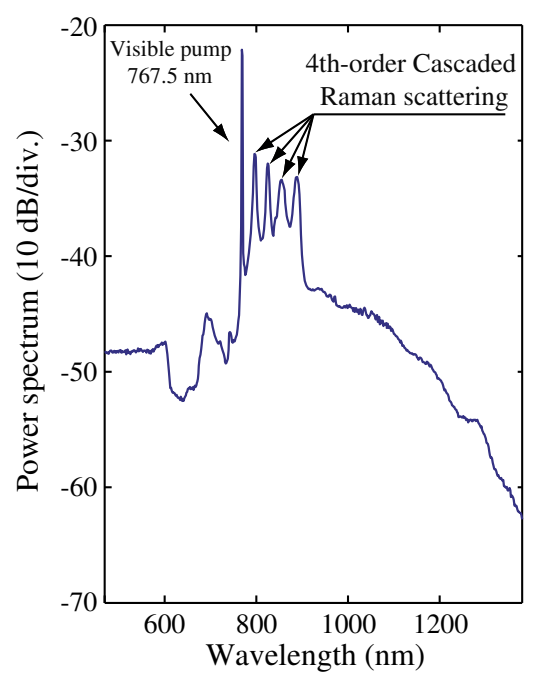

Fig. 5. This output spectrum shows four Raman Stokes order in the normal dispersion regime below the first ZDW.

smoothing of the discrete Raman cascade in the SC visible part and the MI sidebands, as it can be seen in Fig. 6 .

Finally a wide supercontinuum is generated in the two ZDW PCFs for a special coupling of both components in the PCF. The SC spans from $550 \mathrm{~nm}$ to more than $1800 \mathrm{~nm}$ in Fig. 7 which are the lower and upper limits of our spectrum analyzer. Note that the blue and red sides of the SC spectrum appear with a small amplitude because of the low quantum efficiency of the OSA detector at those wavelengths. The SC short wavelength side is well below $550 \mathrm{~nm}$ given that the SC is white light and the red side should probably reach more than $2 \mu \mathrm{m}$. The insets also show that nearly white light SC generation is obtained in the fundamental optical mode. The total SC output mean power was measured to $1.5 \mathrm{~mW}$ and the SC flatness is relatively poor (about $20 \mathrm{~dB}$ ). However, these could easily be improved by separating the two laser beams using a dichroic filter and by matching their beam waists, as done in Ref. [13]. The use of a fibered-PPLN waveguide should also greatly simplify and improve the SC power and flatness.

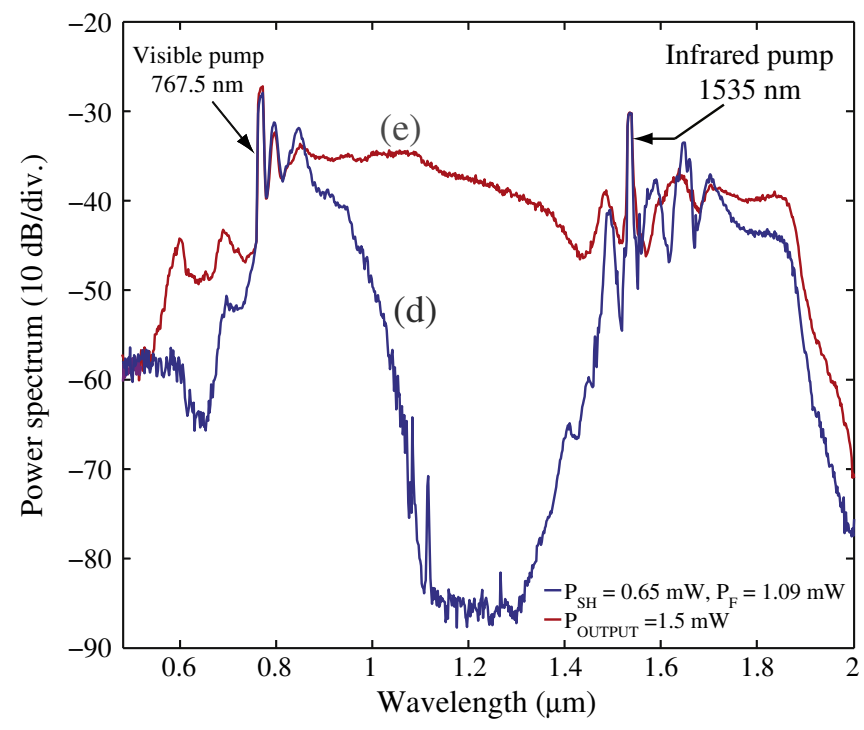

Fig. 6. Output spectra from Fig. 3 associated with d and e horizontal dotted lines showing the merging of the two SCs. $\mathrm{P}_{\mathrm{SH}}$ and $\mathrm{P}_{\mathrm{F}}$ are the estimated mean power of the second harmonic and fundamental components respectively and $\mathrm{P}_{\mathrm{OUTPUT}}$ is the total output power of the most extended supercontinuum. 


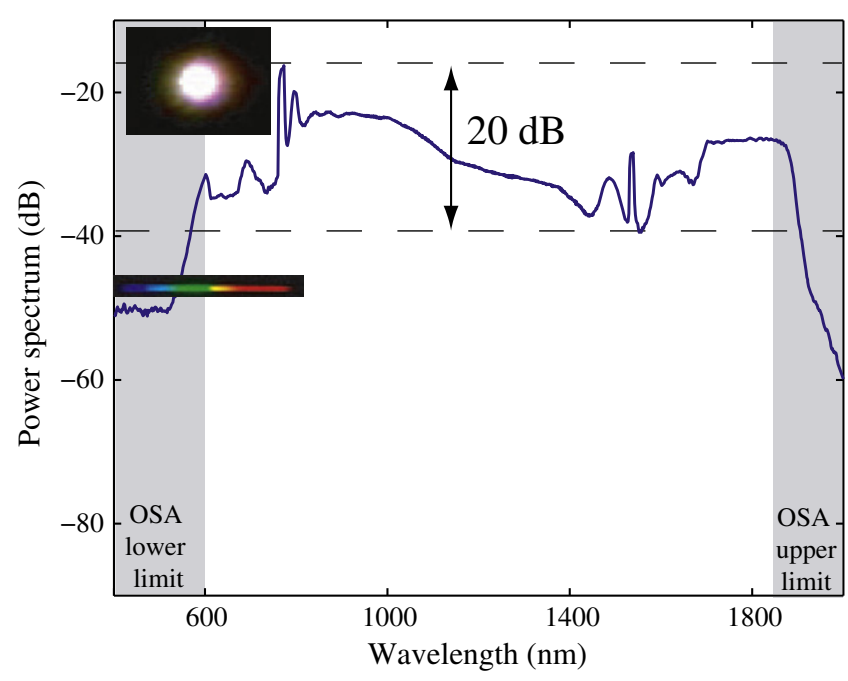

Fig. 7. Supercontinuum generation obtained for maximum coupling efficiency of both the infrared and visible pumps. Insets show the output spatial single-mode behavior and the visible part dispersed by a diffraction grating.

\section{Conclusion and discussion}

In this paper, we experimentally investigated supercontinuum generation by dual nanosecond pumping in a two widely spaced zerodispersion wavelengths of a highly nonlinear photonic crystal fiber. This was achieved by using a compact microchip laser at $1535 \mathrm{~nm}$ and frequency doubled by a PPLN crystal at $767.5 \mathrm{~nm}$. These two wavelengths nearly match the two zero-dispersion wavelengths and generate visible and infrared supercontinuua that merge at high pump powers. The SC shows a spectral extent from $550 \mathrm{~nm}$ to more than $1900 \mathrm{~nm}$. Finally, the pumping scheme that we demonstrated in this work could be advantageously used to pump small-core PCF with ZDW near $770 \mathrm{~nm}$, close to the SHG wavelength, compared to bulky and costly Ti: Sapphire femtosecond laser, for applications such as those requiring commonly incandescent lights [20].

\section{Acknowledgements}

This work was supported financially by the European INTERREG IVA programme (FEDER) and by the Conseil Régional de Franchecomté. The author thanks M. Delqué for numerics and A. Boucon acknowledges the Conseil Général du Doubs for financial support.

\section{References}

[1] J.K. Ranka, R.S. Windeler, A.J. Stentz, Opt. Lett. 25 (2000) 25.

[2] J.M. Dudley, G. Genty, S. Coen, Rev. Mod. Phys. 78 (2006) 1135

[3] J.M. Dudley, J.R. Taylor, Nat. Photonics 3 (2009) 85.

[4] D.V. Skryabin, A.V. Gorbach, Rev. Mod. Phys. 82 (2010) 1287.

[5] N.I. Nikolov, T. Sorensen, O. Bang, J. Opt. Soc. Am. B 20 (2003) 2329

[6] K.M. Hilligsoe, T.V. Andersen, H.N. Paulsen, C.K. Nielsen, K. Malmer, S. Keiding, R. Kristiansen, K.P. Hansen, J.J. Larsen, Opt. Express 12 (2004) 1045.

[7] G. Genty, M. Lethonen, H. Ludvigen, Opt. Express 12 (2004) 3471.

[8] M.H. Frosz, P. Falk, O. Bang, Opt. Express 13 (2005) 6181.

[9] M.L.V. Tse, P. Horak, F. Poletti, N.G.R. Broderick, J.H.V. Price, J.R. Hayes, D.J Richardson, Opt. Express 14 (2006) 4445

[10] A. Mussot, M. Beaugeois, M. Bouazaoui, T. Sylvestre, Opt. Express 15 (2007) 11553.

[11] B. Barviau, B. Kibler, A. Kudlinski, A. Mussot, G. Millot, A. Picozzi, Opt. Express 17 (2009) 7392

[12] P.-A. Champert, V. Couderc, P. Leproux, S. Fvrier, V. Tombelaine, L. Labont, P. Roy, C. Froehly, P. Nrin, Opt. Express 12 (2004) 4366.

[13] T. Schreiber, T.V. Andersen, D. Schimpf, J. Limpert, A. Tnnermann, Opt. Express 13 (2005) 9556.

[14] E. Räikkönen, G. Genty, O. Kimmelma, M. Kaivola, K.P. Hansen, S.C. Buchter, Opt. Express 14 (2006) 7914.

[15] C. Xiong, Z. Chen, W.J. Wadsworth, IEEE J. Ligthwave Technol. 27 (2009) 1638.

[16] G. Genty, M. Lehtonen, H. Ludvigsen, Opt. Lett. 30 (2005) 756.

[17] A. Boucon, D. Alasia, J.-C. Beugnot, G. Mélin, S. Lempereur, A. Fleureau, H. Maillotte J.M. Dudley, T. Sylvestre, IEEE Photonic Technol. Lett. 20 (2008) 842.

[18] D. Taverner, P. Britton, P. Smith, D. Richardson, G. Ross, D. Hanna, Opt. Lett. 23 (1998) 162.

[19] D.V. Skryabin, F. Luan, J.C. Knight, P.S.J. Russell, Science 301 (2003) 1705.

[20] K.P. Hansen, J. Opt. Fiber Commun. Rep. 2 (2005) 226. 